\title{
IR and sub-mm fluxes of SN1987A revisited: when moderate dust masses suffice
}

\author{
A. Sarangi and I. Cherchneff \\ Department Physik, Universität Basel \\ Klingelbergstrasse 82, CH-4056 Basel, Switzerland
}

\begin{abstract}
We model the fluxes in the infrared and submillimeter domain using the dust chemical composition and mass derived from the physico-chemical model of a Type II-P supernova ejecta with stellar progenitor of $19 M_{\odot}$. Our results highlight that the dust mass predicted to rise over time in our chemical models from $10^{-2}$ to $10^{-1} M_{\odot}$ satisfactorily reproduce the infrared and sub millimeter fluxes. They confirm that type II-P SNe are efficient but moderate dust makers in galaxies.
\end{abstract}

Keywords. supernovae: general, dust, infrared: general

\section{Introduction}

Observations in the infrared (IR) and submillimeter (submm) indicate the presence of molecules and dust in the ejecta of type II-P supernova. The mass of dust formed in the ejecta is still highly debated: IR observations indicate small dust mass $\left(10^{-5}\right.$ to $\left.10^{-3} M_{\odot}\right)$ formed before 500 days post-explosion, while submm observations with Herschel reveal large reservoirs of cool dust $\left(10^{-2}\right.$ to $\left.0.7 M_{\odot}\right)$ in supernova remnants. The chemistry and time evolution of the ejecta of a typical type II-P supernova with solar metallicity has been studied (Sarangi \& Cherchneff 2013). The synthesis of dust grain was modelled with a bottom-up approach through formation of molecules and small clusters in the gas phase from day 100 to 2000 post-explosion. The upper limits on dust masses in the ejecta was estimated for different progenitors (Cherchneff \& Sarangi, this volume). We then model the fluxes emitted by dust at different epochs and in different wavelength ranges using the $3 \mathrm{D}$ radiative code MOCASSIN (Ercolano et al. 2007) and reproduce the Spitzer and Herschel data.

\section{Dust mass}

The ejecta parameters correspond to a typical $19 M_{\odot}$ model (Sarangi \& Cherchneff 2013) and the elemental yields are those of Woosley (1988). We model the formation of small dust clusters that include dimers of forsterite $\left(\mathrm{Mg}_{4} \mathrm{Si}_{2} \mathrm{O}_{8}\right)$, silica, carbon $\operatorname{ring}\left(\mathrm{C}_{10}\right)$, alumina, silicon carbide, iron and magnesium sulphide, and metallic clusters of $\mathrm{Fe}, \mathrm{Mg}$ and $\mathrm{Si}$. The cluster masses represent an upper limit to the total dust mass and increases from $10^{-4} M_{\odot}$ at day 400 to $\sim 0.1 M_{\odot}$ at day 2000 post-explosion. The prevalent dust clusters are carbon $\left(0.08 M_{\odot}\right)$, forsterite $\left(0.014 M_{\odot}\right)$ and alumina $\left(6 \times 10^{-3} M_{\odot}\right)$.

\section{Modelling of IR \& submm fluxes from SN1987A}

Based on the dust chemical composition and mass from the model, the dust contribution to the flux at different epochs was assessed. The observed IR flux from SN1987A at 
Table 1. Input parameters used in MOCASSIN to model the IR flux from SN1987A

\begin{tabular}{ccccccc}
\hline $\begin{array}{c}\text { Time } \\
(\text { days })\end{array}$ & $\begin{array}{c}\text { Rin } \\
\left(10^{15} \mathrm{~cm}\right)\end{array}$ & $\begin{array}{c}\text { Rout } \\
\left(10^{15} \mathrm{~cm}\right)\end{array}$ & $\begin{array}{c}\text { Luminosity } \\
\left(L_{\odot}\right)\end{array}$ & $\begin{array}{c}\text { Blackbody } \\
(\mathrm{K})\end{array}$ & $\begin{array}{c}\text { Mass } \\
\left(10^{-2} M_{\odot}\right)\end{array}$ & $\begin{array}{c}\text { Composition } \\
\text { Silicate/Alumina }(\%)\end{array}$ \\
\hline 615 & 9.5 & 95 & $6.7 \mathrm{e} 5$ & 6200 & 1.0 & $99 / 1$ \\
\hline
\end{tabular}

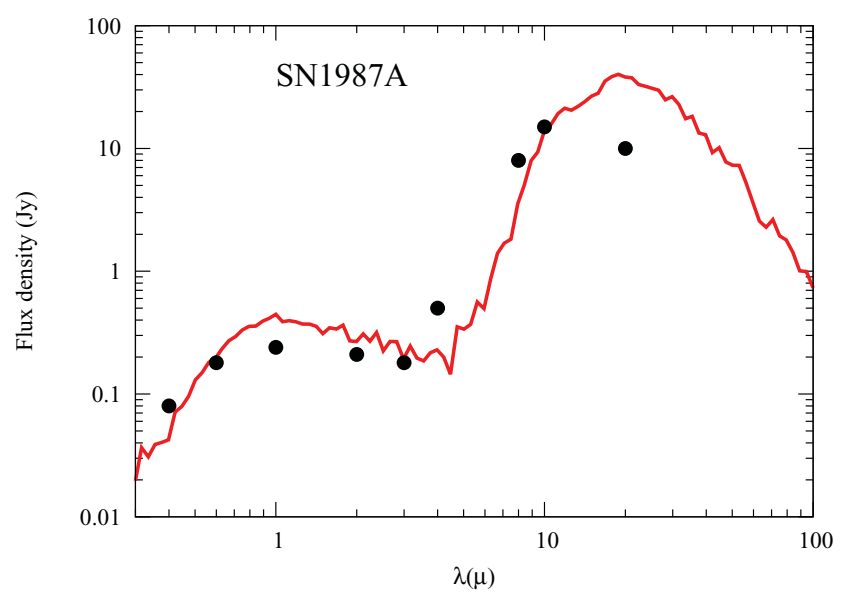

Figure 1. IR flux emitted by our modelled dust composition and mass for a $19 M_{\odot}$ progenitor at day 615 post-explosion. The IR flux data of SN1987A at 615 days are shown.

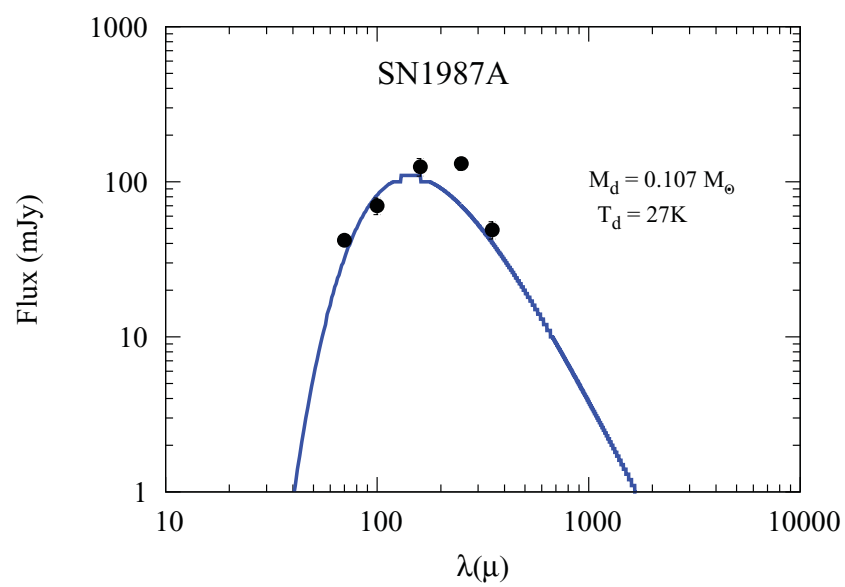

Figure 2. Submm flux emitted by our modelled dust composition and mass for a $19 M_{\odot}$ progenitor. The dust temperature is $27 \mathrm{~K}$. The submm dust flux data from Herschel are also shown.

615 days was modelled with the $3 \mathrm{D}$ radiative transfer code MOCASSIN (Ercolano et al. 2007). A smooth model (i.e., without clumps) with a grain size distribution ranging from 0.005 to $0.05 \mu \mathrm{m}$ was considered. The input parameters are listed in Table 1 . The fitting of the IR data with our modelled dust composition and mass at 615 days is presented in Fig. 1. The submm data of SN1987A from the Herschel space telescope (Matsuura et al. 2011 ) is fitted by the dust composition and mass derived at day 2000 and an absorption coefficient dependant black body fit. The best fit is achieved for a dust temperature of 27 K (Fig. 2). 


\section{Implication}

Our results highlight that a moderate mass of dust $\left(10^{-2}\right.$ to $\left.0.1 M_{\odot}\right)$ is sufficient to reproduce the IR flux of the supernova SN1987A and the submm flux measured in the young remnant. They indicate that type II-P SNe are efficient but moderate dust makers in galaxies.

\section{References}

Ercolano, B., Barlow, M. J., Sugerman, B. E. K., 2007 MNRAS, 375, 753

Matsuura, M., Dwek, E., Meixner, M. et al. 2011 Science, 333, 1258

Sarangi, A. \& Cherchneff, I., 2013 ApJ, submitted

Woosley, S. E, 1988, ApJ, 330, 218 